Article

\title{
Nutrition Composition and Single, 14-Day and 13-Week Repeated Oral Dose Toxicity Studies of the Leaves and Stems of Rubus coreanus Miquel
}

\author{
Ae-Son Om ${ }^{1, *}$, Yu-Na Song ${ }^{1}$, GeonMin Noh ${ }^{2}$, HaengRan Kim ${ }^{2}$ and JeongSook Choe ${ }^{2}$ \\ Received: 26 October 2015 ; Accepted: 31 December 2015 ; Published: 8 January 2016 \\ Academic Editor: Derek J. McPhee \\ 1 Laboratory of Food Safety and Toxicology, Department of Food Science and Nutrition, Hanyang University, \\ Seoul 133-791, Korea; syn0878@gmail.com \\ 2 Functional Food and Nutrition Division, Department of Agrofood Resources, National Academy of \\ Agricultural Science, Jeonbuk 565-851, Korea; team0708@korea.kr (G.M.N.); hrrkim@korea.kr (H.R.K.); \\ swany@korea.kr (J.S.C.) \\ * Correspondence: aesonom@hanyang.ac.kr; Tel.: +82-2-2220-1203; Fax: +82-2-2220-1856
}

\begin{abstract}
The leaves and stems of the plant Rubus coreanus Miquel (RCMLS) are rich in vitamins, minerals and phytochemicals which have antioxidant, anti-hemolytic, anti-inflammatory, anti-fatigue and anti-cancer effects. However, RCMLS is not included in the Korean Food Standards Codex due to the lack of safety assurance concerning RCMLS. We evaluated single and repeated oral dose toxicity of RCMLS in Sprague-Dawley rats. RCMLS did not induce any significant toxicological changes in both male and female rats at a single doses of $2500 \mathrm{mg} / \mathrm{kg} /$ day. Repeated oral dose toxicity studies showed no adverse effects in clinical signs, body weight, food consumption, ophthalmic examination, urinalysis, hematology, serum biochemistry, necropsy findings, organ weight, and histopathology at doses of 625, 1250, and $2500 \mathrm{mg} / \mathrm{kg} /$ day. The LD 50 and LOAEL of RCMLS might be over $2500 \mathrm{mg} / \mathrm{kg}$ body weight/day and no target organs were identified. Therefore, this study revealed that single and repeated oral doses of RCMLS are safe.
\end{abstract}

Keywords: Rubus coreanus Miquel leaves and stems; single dose toxicity; repeated dose toxicity; nutrition composition

\section{Introduction}

Rubus coreanus Miquel (R. coreanus) is a deciduous broadleaf shrub of the Rosaceae family that originates from Korea and China. The unripe fruit of $R$. coreanus, commonly called bokbunja in Korea, contains various bioactive phenolic compounds such as flavonoids, anthocyanins, tannins, quercetin, as well as minerals, vitamins, etc. [1]. The unripe fruits of $R$. coreanus are used in traditional Korean herbal medicine to treat diabetes, spermatorrhea, enuresis, asthma and allergy-related illnesses [2,3]. With their high anthocyanin content the ripe fruits of $R$. coreanus are darker in color compared to other berries. They have an abundance of phenolic compounds such as ellagic acid, gallic acid, cinnamic acid, protocatechuic acid, sangiin H-4, sanguiin H-6, 23-hydroxytormentic acid, and nigaichgoside F [4-7]. They are reported to have antioxidant, antihemolytic, anticancer, anti-inflammatory, antibacterial, and anti-fatigue effects [8-18]. There is a general interest in identifying natural bioactive compounds, because these compounds have functional benefits such as regulating apoptosis in cancer and healthy cells [19].

Meanwhile, the leaves of $R$. coreanus are rich in tannins (ellagic acid, sanguiin $\mathrm{H}-5$ ) and flavonoids (kaemferol, quercetin, quercetin 3-O- $\beta$-D-glucuronide-sodium salt, quercetin 3-O- $\beta$-D-glucuronidesodium carboxylate) [20]. The stems of $R$. coreanus have epicatechin, catechin, procyanidin B-4 and sanguiin H-4 as well [21]. Moreover, the extract of R. coreanus leaves and stems (RCMLS) have 
been reported to have HMG-CoA reductase activity, nitric oxide generating, angiotensin converting enzyme, protein expression suppressing and endothelial NOS increasing effects, which are important factors in the cholesterol biosynthesis process $[22,23]$. $R$. coreanus has been consumed since the old times and its fruits, leaves and stems are known to possess physiological and pharmacological effects. RCMLS is also widely consumed in side dishes, teas, drinks or medical herbs in Korean folk medicine, but it is still not registered as a food material in the Korean Food Standards Codex. For food material registration, safety data obtained though toxicological testing is essential, but to date there are no safety studies on RCMLS. Therefore, this study aimed to provide a basis for the nutrition composition of RCMLS and to evaluate its single, 14 days and 13 weeks repeated oral dose toxicity in Sprague-Dawley rats.

\section{Results and Discussion}

\subsection{Nutrition Compositon}

The proximate composition, minerals and vitamins contents of RCMLS are shown in Table 1. Carbohydrates $(76.81 \%)$ were the highest in content, which includes $66.42 \%$ fiber. Crude protein, fat, and moisture were $10.07 \%, 2.20 \%$, and $5.57 \%$ on a dry weight basis, respectively.

Table 1. Nutrition compositions of the leaves and stems of Rubus coreanus Miquel.

\begin{tabular}{ccccc}
\hline Nutrient & Ingredient & Content & Ingredient & Content \\
\hline \multirow{2}{*}{$\begin{array}{c}\text { Proximate composition } \\
(\%)\end{array}$} & Moisture & 5.75 & Carbohydrate & 76.81 \\
& Ash & 5.17 & Crude fat & 2.20 \\
& Crude protein & 10.07 & & \\
\hline & $\mathrm{Ca}$ & 810.92 & $\mathrm{~K}$ & 1463.96 \\
& $\mathrm{Mg}$ & 263.28 & $\mathrm{Cr}$ & 0.66 \\
Minerals (mg/100 g) & $\mathrm{Fe}$ & 13.57 & $\mathrm{Se}$ & $7.06 \mu \mathrm{g} / 100 \mathrm{~g}$ \\
& $\mathrm{Zn}$ & 7.21 & $\mathrm{I}$ & $20.88 \mu \mathrm{g} / 100 \mathrm{~g}$ \\
& $\mathrm{Cu}$ & 0.53 & $\mathrm{Mo}$ & $37.02 \mu \mathrm{g} / 100 \mathrm{~g}$ \\
& $\mathrm{Mn}$ & 30.37 & & 7.93 \\
& $\mathrm{~B}_{1}$ & 0.04 & $\mathrm{E}$ & 0.82 \\
& $\mathrm{~B}_{2}$ & 0.10 & $\mathrm{~K}$ & $0.51 \mathrm{mg} \mathrm{NE} / 100 \mathrm{~g}$ \\
\hline
\end{tabular}
The contents were calculated by dry weight basis \% (unit: \%, weight/weight); ${ }^{1}$ Carbohydrate includes $66.42 \%$
dietary fiber.

In mineral contents, $\mathrm{K}(1463.96 \mathrm{mg} / 100 \mathrm{~g}), \mathrm{Ca}(810.92 \mathrm{mg} / 100 \mathrm{~g})$, and $\mathrm{Mg}(263.28 \mathrm{mg} / 100 \mathrm{~g})$ levels were high and $\mathrm{Mn}(30.37 \mathrm{mg} / 100 \mathrm{~g}), \mathrm{Fe}(13.57 \mathrm{mg} / 100 \mathrm{~g}), \mathrm{Zn}(7.21 \mathrm{mg} / 100 \mathrm{~g})$ were detectable. In vitamins, vitamin $\mathrm{E}$ was the highest at $7.93 \mathrm{mg} \alpha-\mathrm{TE} / 100 \mathrm{~g}$ and the order of the remaining ones was vitamin $\mathrm{K}>$ niacin $>$ vitamin $\mathrm{B}_{6}>$ vitamin $\mathrm{B}_{2}>$ vitamin $\mathrm{D}>$ vitamin $\mathrm{B}_{1}$.

Kim et al. [12] reported that fruits of $R$. coreanus fall in the range of $8 \%-10 \%$ moisture, $4 \%-5 \%$ crude protein, $3 \%-5 \%$ crude fat, $4 \%-7 \%$ ash, $12 \%-56 \%$ free sugar, and $11 \%-37 \%$ fiber on a dry weight basis. The mineral contents of $R$. coreanus were K (10,381-21,026 ppm), Ca (4976-11,489 ppm), Mg (2789-4446 ppm), P (2364-3425 ppm), Mg (151-200 ppm), Fe (32-37 ppm), Zn (15 ppm), Na (9-18 ppm) and $\mathrm{Cu}(7-8 \mathrm{ppm})$ on a dry weight basis. According to previous studies, the proximate composition of RCMLS is similar to that of fruits. Jung et al. [24] studied the proximate composition of each RCMLS component. The leaves showed $9.72 \%$ moisture, $1.75 \%$ crude protein and $1.75 \%$ crude fat and the stems showed $4.22 \%, 1.32 \%$ and $0.87 \%$, respectively. However, research on the vitamin and fatty acid composition of RCMLS is nonexistent.

Table 2 lists the fatty acid composition of the RCMLS. $\alpha$-linolenic acid was the highest component at $30.48 \%$, followed by palmitic acid at $23.68 \%$ and linoleic acid at $15.51 \%$ on a dry weight basis. Total 
saturated fatty acid (SFA) was 39.32\%, while monounsaturated fatty acid (MUFA) and polyunsaturated fatty acid (PUFA) were $4.21 \%$ and $46.72 \%$, respectively. Thus, RCMLS contained more unsaturated fatty acid (UFA) than SFA. Meanwhile, Lee et al. [25] reported linoleic acid in the fatty acid composition of ripe fruits of $R$. coreanus was the highest at 3.573\% and the ranking was linolenic acid $>$ oleic acid $>$ palmitic acid $>$ arachidic acid $>$ myristic acid $>$ palmitoleic acid $>$ stearic acid $>$ caproic acid, and PUFA $(70.78 \%)$ was higher than SFA $(11.17 \%)$. The data in this study is thus not in accordance with the study conducted by Lee et al. [25] but UFA was higher than SFA regardless of whether fruits, leaves and stems were studied.

It is hard to compare the absolute content of the mixture of RCMLS as there are no study results analyzing the nutritional composition of the mixture. However, when compared to the previous studies [24] separately analyzing the leaves and stems, the mixture of RCMLS can be estimated to have similar ingredients.

Table 2. Fatty acid compositions of the leaves and stems of Rubus coreanus Miquel.

\begin{tabular}{|c|c|c|c|}
\hline Fatty Acid & Content & Fatty Acid & Content \\
\hline Lauric acid & 1.64 & Heneicosanoic acid & 0.49 \\
\hline Myristic acid & 1.01 & Behenic acid & 3.40 \\
\hline Palmitic acid & 23.68 & Arachidonic acid & 0.73 \\
\hline Heptadecanoic acid & 0.88 & Lignoceric acid & 3.41 \\
\hline Elaidic acid & 0.53 & $\Sigma$ SFA & 39.32 \\
\hline Oleic acid & 4.21 & $\Sigma$ MUFA & 4.21 \\
\hline Linoleic acid & 15.51 & $\Sigma$ PUFA & 46.72 \\
\hline Arachidic acid & 4.81 & $\Sigma$ UFA & 50.93 \\
\hline$\alpha$-Linolenic acid & 30.48 & & \\
\hline
\end{tabular}

The contents were calculated by dry weight basis (unit: \%, weight/weight); Values are expressed as the area percentage of total fatty acid; Abbreviation; SFA: Saturated fatty acid, MUFA: Monounsaturated fatty acid, PUFA: Polyunsaturated fatty acid, UFA: Unsaturated fatty acid.

\subsection{Single Oral Dose Toxicity Study}

To investigate the toxicity and determine the lethal dose $\left(\mathrm{LD}_{50}\right)$, RCMLS was administrated orally to male and female Sprague-Dawley (SD) rats at single doses of 0 (vehicle control) and $2500 \mathrm{mg} / \mathrm{kg}$. During the 14 days experimental period, mortality, clinical signs, body weight and necropsy results were observed. No animal deaths or abnormal clinical signs were observed. In addition, there were no significant changes in body weight in the RCMLS-treated groups compared with the vehicle control group. No abnormalities were found during necropsy. Therefore, $\mathrm{LD}_{50}$ of RCMLS can be estimated to be over $2500 \mathrm{mg} / \mathrm{kg}$ body weight (b.w.).

\subsection{Days Repeated Oral Dose Toxicity Study}

To determine the dosage of RCMLS for the 13 weeks repeated oral dose toxicity tests, male and female SD rats were orally fed with RCMLS for 14 days at doses of 0 (vehicle control), 625, 1250 and $2500 \mathrm{mg} / \mathrm{kg} /$ day. During the experimental period, mortality, clinical signs, body weight, food consumption, ophthalmic examination, urinalysis, hematology, serum biochemistry, necropsy, organ weight and histopathology were observed.

In the results, no animal deaths or abnormal clinical signs related to the administration of RCMLS were observed (Table 3). In addition, there were no significant body weight changes in RCMLS-treated groups compared with the vehicle control group (Table 4). Ophthalmic examination showed no abnormality in any test rats. Urinalysis and urine color observation showed no significant difference between male and female rats. Hematology tests showed no significant change caused by feeding RCMLS (Table 6).

Serum biochemistry test showed that $\mathrm{K}^{+}$decreased in the males of the $2500 \mathrm{mg} / \mathrm{kg} /$ day group and $\mathrm{Ca}^{2+}$ and $\mathrm{Na}^{+}$increased and $\mathrm{Cl}^{-}$decreased in the females of the $2500 \mathrm{mg} / \mathrm{kg} /$ day group (Table 7). 
However, the range was very slight and within the biological change range [26]. Therefore, it was not considered toxicologically significant.

Table 3. Mortality and clinical sign of 14 days repeated oral dose and 13 weeks repeated oral dose toxicity studies of the leaves and stems of Rubus coreanus Miquel (\%).

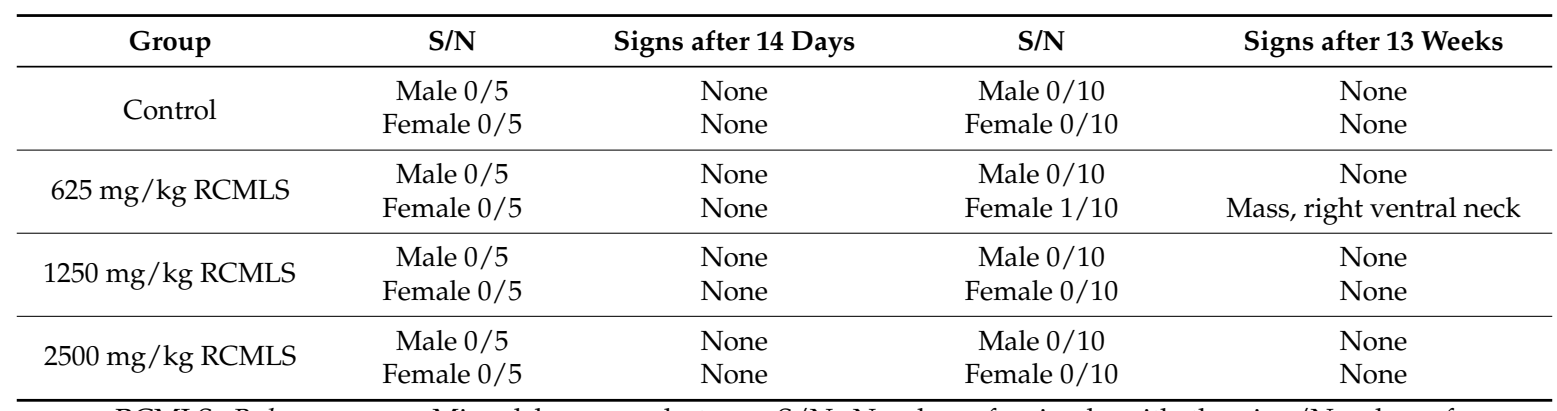

RCMLS: Rubus coreanus Miquel leaves and stems, S/N: Number of animals with the sign/Number of animals examined.

Table 4. Body weight of male and female rats in 14 days repeated oral dose toxicity study of the leaves and stems of Rubus coreanus Miquel.

\begin{tabular}{cccccc}
\hline \multirow{2}{*}{ Group } & Sex & \multicolumn{3}{c}{ Body Weight (g) } & Weight Gain \\
\cline { 3 - 5 } & & Day 0 & Day 7 & Day 14 & \\
\hline \multirow{2}{*}{ Control } & Male & $194.17 \pm 5.37$ & $258.98 \pm 9.15$ & $294.64 \pm 17.73$ & 52.6 \\
& Female & $163.66 \pm 6.81$ & $189.22 \pm 14.95$ & $217.95 \pm 20.84$ & 33.1 \\
\hline $625 \mathrm{mg} / \mathrm{kg}$ & Male & $194.83 \pm 8.09$ & $261.06 \pm 8.46$ & $311.47 \pm 11.27$ & 60.9 \\
RCMLS & Female & $161.21 \pm 5.49$ & $191.96 \pm 10.10$ & $214.23 \pm 9.78$ & 33.9 \\
\hline $1250 \mathrm{mg} / \mathrm{kg}$ & Male & $195.53 \pm 6.02$ & $256.15 \pm 10.90$ & $296.68 \pm 13.79$ & 52.8 \\
RCMLS & Female & $160.03 \pm 7.09$ & $190.03 \pm 8.94$ & $213.64 \pm 7.89$ & 33.5 \\
\hline $2500 \mathrm{mg} / \mathrm{kg}$ & Male & $192.78 \pm 6.59$ & $243.43 \pm 11.13$ & $291.00 \pm 18.51$ & 51.0 \\
RCMLS & Female & $163.08 \pm 6.62$ & $187.52 \pm 9.01$ & $207.01 \pm 10.04$ & 27.0 \\
\hline
\end{tabular}

Data are presented as mean \pm standard deviation; RCMLS: Rubus coreanus Miquel leaves and stems.

Absolute and relative organ weight showed no significant change in both male and females of all test groups (Tables 9 and 10). Necropsy and histopathology test results showed miniaturized bilateral testes in the male of the $1250 \mathrm{mg} / \mathrm{kg}$ / day group that were confirmed as seminiferous tubules degeneration and the enlarged spleen in a female of the $2500 \mathrm{mg} / \mathrm{kg}$ / day group were confirmed to be increased cellularity of white pulp. However, it was not considered toxicologically significant because it was an isolated case and a natural occurrence.

In conclusion, when RCMLS was orally administered repeatedly for 14 days to SD rats, no systemic toxicological change in relation to RCMLS was observed. Therefore, setting a dosage as high as $2500 \mathrm{mg} / \mathrm{kg} /$ day in the subsequent 13 weeks repeated administration test seems appropriate.

\subsection{Weeks Repeated Oral Dose Toxicity Study}

\subsubsection{Mortality, Clinical Signs, Body Weight and Food Consumption}

There were no animal deaths during the experimental period, but a female of the $625 \mathrm{mg} / \mathrm{kg} /$ day group showed a subcutaneous mass in the right dorsal neck after 13 weeks of RCMLS administration until nucropsy (Table 3). However it was concluded as unrelated to RCMLS administration by necropsy and histopathology examination

Weight changes showed that on the 11-13 weeks, the weight of males in the $2500 \mathrm{mg} / \mathrm{kg} /$ day group decreased significantly compared to the vehicle control group (Table 5). These findings 
are concluded to be an effect of RCMLS but are within biological fluctuation range and other test items showed no support for adverse effect of RCMLS. Therefore, it was not considered toxicologically significant.

Table 5. Body weight of male and female rats in 13 weeks repeated oral dose toxicity study of the leaves and stems of Rubus coreanus Miquel.

\begin{tabular}{cccccc}
\hline \multirow{2}{*}{ Group } & Sex & \multicolumn{3}{c}{ Body Weight (g) } & Weight Gain \\
\cline { 3 - 5 } & & Week 0 & Week 7 & Week 13 & \\
\hline \multirow{2}{*}{ Control } & Male & $188.01 \pm 7.26$ & $477.50 \pm 37.41$ & $579.16 \pm 56.03$ & 208.0 \\
& Female & $142.95 \pm 7.05$ & $269.69 \pm 22.30$ & $313.66 \pm 26.59$ & 119.4 \\
\hline $625 \mathrm{mg} / \mathrm{kg}$ & Male & $189.79 \pm 6.70$ & $495.16 \pm 35.13$ & $611.26 \pm 43.19$ & 222.1 \\
$\mathrm{RCMLS}$ & Female & $142.39 \pm 5.09$ & $269.05 \pm 26.70$ & $311.62 \pm 41.69$ & 118.9 \\
\hline $1250 \mathrm{mg} / \mathrm{kg}$ & Male & $190.20 \pm 6.76$ & $500.33 \pm 45.79$ & $614.66 \pm 47.94$ & 123.2 \\
RCMLS & Female & $144.22 \pm 7.73$ & $274.73 \pm 29.00$ & $321.73 \pm 39.22$ & 123.1 \\
\hline $2500 \mathrm{mg} / \mathrm{kg}$ & Male & $184.96 \pm 12.72$ & $445.67 \pm 44.60$ & $533.00 \pm 54.44^{* *}$ & $188.2 * *$ \\
RCMLS & Female & $141.71 \pm 6.27$ & $260.15 \pm 22.28$ & $296.31 \pm 24.78$ & 109.1 \\
\hline
\end{tabular}

Data are presented as mean \pm standard deviation; ** Significantly different from corresponding control value at $p<0.01$; RCMLS: Rubus coreanus Miquel leaves and stems.

Food consumption measurement results showed 6-8 weeks period food consumption in the male of the $2500 \mathrm{mg} / \mathrm{kg}$ group and 12 weeks period in the female of the $2500 \mathrm{mg} / \mathrm{kg}$ group decreased significantly compared to the vehicle control group. However, these changes were transient and appeared to be unrelated to the doses or to the treatment with RCMLS.

\subsubsection{Ophthalmic Examination, Urinalysis, Hematology and Serum Biochemistry}

No abnormalities were found in any groups in the ophthalmic examination and urinalysis.

Hematopoietic parameters are some of the most sensitive ones used to assess the toxicity of drugs in humans and animals, and a blood profile usually gives vital information on the response of the body to injury or stress $[27,28]$. In the present study, the hematology test results showed a significant decrease in mean platelet volume (MPV) in the males of the $2500 \mathrm{mg} / \mathrm{kg} /$ day group compared to the vehicle control group. Additionally, neutrophil (NE) and percent of neutrophil (NEP) in the females of the $625 \mathrm{mg} / \mathrm{kg} /$ day group significantly increased, whereas the percent of lymphocyte (LYP) decreased significantly. However, the difference was slight and within the biological fluctuation range [26], with no dose-dependency. Thus, it was concluded that RCMLS did not influence it.

Serum biochemistry result showed dose-dependency in total bilirubin and $\mathrm{Mg}^{2+}$ of the females of the $2500 \mathrm{mg} / \mathrm{kg} /$ day group and it significantly decreased when compared to the vehicle control group. $\mathrm{Na}^{+}$in females of the 1250 and $2500 \mathrm{mg} / \mathrm{kg} /$ day group decreased significantly in a dose dependent manner (Table 8). But the changes were considered incidental, because they were very slight changes, not sex or dose-related, and unaccompanied by any correlative finding.

\subsubsection{Autopsy, Organ Weights and Histopathology}

One case of cyst in the pituitary gland was observed in a male of the vehicle control group and one case of a mass in the right subcutaneous neck was observed in a female of the $625 \mathrm{mg} / \mathrm{kg} /$ day group. However, considering the histopathology test results, these findings were non dose-dependent and a natural occurrence or temporary and therefore, it was not considered toxicologically significant.

Absolute liver weights in the males of the $2500 \mathrm{mg} / \mathrm{kg}$ / day group, absolute right ovary weights in the females of the $2500 \mathrm{mg} / \mathrm{kg} /$ day group, relative both testes weights in males of the 625 and $1250 \mathrm{mg} / \mathrm{kg} /$ day group, and relative left adrenal gland weights in the males of the $1250 \mathrm{mg} / \mathrm{kg} /$ day group, decreased significantly when compared with those in the vehicle control group (Tables 9 and 10). 
Table 6. Hematological values of male and female rats in 14 days repeated oral dose and 13 weeks repeated oral dose toxicity studies of the leaves and stems of Rubus coreanus Miquel (\%).

\begin{tabular}{|c|c|c|c|c|c|c|c|c|c|}
\hline \multirow{2}{*}{ Group } & \multirow{2}{*}{ Sex } & \multicolumn{2}{|c|}{ HGB (g/dL) } & \multicolumn{2}{|c|}{ HCT (\%) } & \multicolumn{2}{|c|}{ WBC $(\mathrm{K} / \mu \mathrm{L})$} & \multicolumn{2}{|c|}{ Platelet $(\mathrm{K} / \mu \mathrm{L})$} \\
\hline & & 14 Days & 13 Weeks & 14 Days & 13 Weeks & 14 Days & 13 Weeks & 14 Days & 13 Weeks \\
\hline \multirow{2}{*}{ Control } & Male & $14.7 \pm 0.8$ & $14.6 \pm 0.9$ & $45.5 \pm 2.3$ & $45.4 \pm 2.5$ & $7.01 \pm 1.48$ & $11.21 \pm 3.49$ & $1217 \pm 90$ & $990 \pm 203$ \\
\hline & Female & $14.8 \pm 1.0$ & $15.1 \pm 0.5$ & $45.2 \pm 2.5$ & $45.9 \pm 1.6$ & $7.62 \pm 2.66$ & $6.83 \pm 2.32$ & $1310 \pm 129$ & $841 \pm 98$ \\
\hline \multirow{2}{*}{$\begin{array}{c}625 \mathrm{mg} / \mathrm{kg} \\
\text { RCMLS }\end{array}$} & Male & $14.1 \pm 0.4$ & $14.7 \pm 0.6$ & $44.3 \pm 1.2$ & $45.9 \pm 1.9$ & $8.22 \pm 1.60$ & $11.07 \pm 2.85$ & $1401 \pm 117$ & $1112 \pm 73$ \\
\hline & Female & $15.4 \pm 0.6$ & $15.2 \pm 0.5$ & $46.5 \pm 1.8$ & $46.1 \pm 1.2$ & $10.77 \pm 4.36$ & $7.35 \pm 1.29$ & $1276 \pm 197$ & $947 \pm 121$ \\
\hline \multirow{2}{*}{$\begin{array}{c}1250 \mathrm{mg} / \mathrm{kg} \\
\text { RCMLS }\end{array}$} & Male & $14.3 \pm 0.6$ & $14.6 \pm 0.4$ & $44.7 \pm 1.5$ & $45.2 \pm 1.4$ & $8.15 \pm 1.27$ & $11.90 \pm 2.00$ & $1243 \pm 113$ & $1062 \pm 143$ \\
\hline & Female & $15.2 \pm 0.4$ & $15.1 \pm 0.5$ & $45.7 \pm 1.3$ & $45.7 \pm 1.5$ & $8.63 \pm 1.27$ & $6.85 \pm 1.78$ & $1231 \pm 102$ & $925 \pm 143$ \\
\hline \multirow{2}{*}{$\begin{array}{c}2500 \mathrm{mg} / \mathrm{kg} \\
\text { RCMLS }\end{array}$} & Male & $14.0 \pm 0.9$ & $15.1 \pm 0.3$ & $43.6 \pm 3.2$ & $47.3 \pm 0.8$ & $8.55 \pm 1.42$ & $9.72 \pm 1.90$ & $1203 \pm 146$ & $1102 \pm 151$ \\
\hline & Female & $14.8 \pm 0.6$ & $15.2 \pm 0.6$ & $44.8 \pm 1.4$ & $45.9 \pm 1.6$ & $7.88 \pm 0.93$ & $6.87 \pm 1.96$ & $1314 \pm 295$ & $965 \pm 115$ \\
\hline \multirow{2}{*}{ Group } & \multirow{2}{*}{ Sex } & \multicolumn{2}{|c|}{ MPV (fL) } & \multicolumn{2}{|c|}{$\mathrm{NE}(\mathrm{K} / \mu \mathrm{L})$} & \multicolumn{2}{|c|}{ NEP (\%) } & \multicolumn{2}{|c|}{ LYP (\%) } \\
\hline & & 14 Days & 13 Weeks & 14 Days & 13 Weeks & 14 Days & 13 Weeks & 14 Days & 13 Weeks \\
\hline \multirow{2}{*}{ Control } & Male & $5.5 \pm 0.8$ & $4.7 \pm 0.3$ & $1.04 \pm 0.30$ & $2.16 \pm 1.17$ & $15.6 \pm 6.6$ & $19.0 \pm 7.5$ & $79.6 \pm 7.3$ & $76.1 \pm 8.5$ \\
\hline & Female & $5.6 \pm 0.5$ & $4.6 \pm 0.2$ & $0.75 \pm 0.18$ & $0.99 \pm 0.34$ & $10.7 \pm 3.7$ & $15.0 \pm 5.0$ & $85.4 \pm 3.9$ & $79.9 \pm 5.0$ \\
\hline \multirow{2}{*}{$\begin{array}{c}625 \mathrm{mg} / \mathrm{kg} \\
\text { RCMLS }\end{array}$} & Male & $5.7 \pm 1.2$ & $4.6 \pm 0.1$ & $1.23 \pm 0.24$ & $2.00 \pm 1.23$ & $15.5 \pm 4.1$ & $17.6 \pm 9.1$ & $80.6 \pm 3.9$ & $77.8 \pm 9.9$ \\
\hline & Female & $4.8 \pm 0.5$ & $4.5 \pm 0.3$ & $0.84 \pm 0.48$ & $1.55 \pm 0.58^{* *}$ & $8.5 \pm 4.3$ & $20.7 \pm 5.6^{* *}$ & $87.2 \pm 3.8$ & $74.1 \pm 6.0$ ** \\
\hline \multirow{2}{*}{$\begin{array}{c}1250 \mathrm{mg} / \mathrm{kg} \\
\text { RCMLS }\end{array}$} & Male & $5.4 \pm 0.8$ & $4.7 \pm 0.2$ & $1.08 \pm 0.25$ & $2.06 \pm 0.60$ & $13.3 \pm 2.9$ & $17.4 \pm 4.5$ & $82.6 \pm 2.6$ & $77.8 \pm 4.6$ \\
\hline & Female & $5.2 \pm 0.5$ & $4.6 \pm 0.2$ & $0.86 \pm 0.17$ & $0.79 \pm 0.30$ & $10.1 \pm 2.3$ & $12.3 \pm 5.3$ & $86.2 \pm 3.0$ & $82.3 \pm 5.8$ \\
\hline \multirow{2}{*}{$\begin{array}{c}2500 \mathrm{mg} / \mathrm{kg} \\
\text { RCMLS }\end{array}$} & Male & $5.8 \pm 1.0$ & $4.5^{*} \pm 0.1$ & $1.24 \pm 0.67$ & $1.81 \pm 0.68$ & $14.4 \pm 6.7$ & $19.0 \pm 7.5$ & $81.6 \pm 7.8$ & $76.4 \pm 7.6$ \\
\hline & Female & $4.9 \pm 0.7$ & $4.5 \pm 0.2$ & $0.82 \pm 0.16$ & $0.95 \pm 0.39$ & $10.4 \pm 1.5$ & $14.2 \pm 4.6$ & $85.1 \pm 1.3$ & $81.7 \pm 4.7$ \\
\hline
\end{tabular}

Data are presented as mean \pm standard deviation; ** Significantly different from corresponding control value at $p<0.01$; RCMLS: Rubus coreanus Miquel leaves and stems, HCT:

hematocrit, HGB: hemoglobin, WBC: white blood corpuscles, MPV: mean platelet volume, NE: neutrophil, NEP; percent of neutrophil, LYP: percent of lymphocyte. 
Table 7. Serum biochemistry in 14 days repeated oral dose toxicity study of the leaves and stems of Rubus coreanus Miquel.

\begin{tabular}{|c|c|c|c|c|}
\hline Parameter & Control & $\begin{array}{c}625 \mathrm{mg} / \mathrm{kg} \\
\mathrm{RCMLS}\end{array}$ & $\begin{array}{c}1250 \mathrm{mg} / \mathrm{kg} \\
\text { RCMLS }\end{array}$ & $\begin{array}{c}2500 \mathrm{mg} / \mathrm{kg} \\
\text { RCMLS }\end{array}$ \\
\hline \multicolumn{5}{|l|}{ Male } \\
\hline Glucose (mg/dL) & $128 \pm 29$ & $128 \pm 17$ & $145 \pm 18$ & $158 \pm 8$ \\
\hline BUN (mg/dL) & $13.1 \pm 1.6$ & $12.5 \pm 1.1$ & $14.2 \pm 1.6$ & $12.1 \pm 2.5$ \\
\hline Creatinine $(\mathrm{mg} / \mathrm{dL})$ & $0.38 \pm 0.04$ & $0.32 \pm 0.05$ & $0.39 \pm 0.04$ & $0.35 \pm 0.03$ \\
\hline Total protein $(\mathrm{g} / \mathrm{dL})$ & $6.0 \pm 0.4$ & $6.0 \pm 0.2$ & $5.9 \pm 0.2$ & $5.7 \pm 0.2$ \\
\hline Albumin (g/dL) & $2.4 \pm 0.2$ & $2.3 \pm 0.2$ & $2.3 \pm 0.1$ & $2.3 \pm 0.2$ \\
\hline $\mathrm{A} / \mathrm{G}$ ratio & $0.66 \pm 0.03$ & $0.62 \pm 0.05$ & $0.65 \pm 0.04$ & $0.65 \pm 0.05$ \\
\hline Total bilirubin (mg/dL) & $0.01 \pm 0.01$ & $0.02 \pm 0.01$ & $0.01 \pm 0.01$ & $0.00 \pm 0.01$ \\
\hline $\mathrm{LDH}(\mathrm{IU} / \mathrm{L})$ & $1706 \pm 631$ & $1777 \pm 923$ & $1754 \pm 689$ & $1793 \pm 761$ \\
\hline Cholesterol (mg/dL) & $62 \pm 7$ & $68 \pm 16$ & $73 \pm 14$ & $68 \pm 16$ \\
\hline Triglycerides (mg/dL) & $28 \pm 7$ & $42 \pm 14$ & $34 \pm 14$ & $35 \pm 15$ \\
\hline $\operatorname{AST}(\mathrm{U} / \mathrm{L})$ & $158 \pm 28$ & $166 \pm 48$ & $167 \pm 43$ & $165 \pm 36$ \\
\hline $\operatorname{ALT}(\mathrm{U} / \mathrm{L})$ & $43 \pm 4$ & $39 \pm 8$ & $44 \pm 6$ & $44 \pm 6$ \\
\hline $\operatorname{ALP}(\mathrm{U} / \mathrm{L})$ & $669 \pm 114$ & $598 \pm 126$ & $654 \pm 108$ & $761 \pm 59$ \\
\hline $\operatorname{Mg}(\mathrm{mg} / \mathrm{dL})$ & $2.9 \pm 0.3$ & $2.8 \pm 0.1$ & $2.9 \pm 0.1$ & $2.7 \pm 0.1$ \\
\hline $\mathrm{Ca}(\mathrm{mg} / \mathrm{dL})$ & $10.0 \pm 0.2$ & $9.9 \pm 0.1$ & $9.7 \pm 0.4$ & $9.7 \pm 0.3$ \\
\hline $\mathrm{Na}(\mathrm{mmol} / \mathrm{L})$ & $139 \pm 3$ & $138 \pm 2$ & $139 \pm 4$ & $137 \pm 3$ \\
\hline $\begin{array}{c}\mathrm{K}(\mathrm{mmol} / \mathrm{L}) \\
\text { Female }\end{array}$ & $6.1 \pm 0.4$ & $5.9 \pm 0.2$ & $5.8 \pm 0.3$ & $5.4 \pm 0.4 *$ \\
\hline Glucose (mg/dL) & $184 \pm 34$ & $198 \pm 36$ & $193 \pm 23$ & $197 \pm 26$ \\
\hline BUN (mg/dL) & $14.8 \pm 2.0$ & $14.2 \pm 2.4$ & $13.9 \pm 2.8$ & $14.7 \pm 1.7$ \\
\hline Creatinine $(\mathrm{mg} / \mathrm{dL})$ & $0.58 \pm 0.11$ & $0.60 \pm 0.13$ & $0.59 \pm 0.11$ & $0.53 \pm 0.13$ \\
\hline Total protein $(\mathrm{g} / \mathrm{dL})$ & $6.7 \pm 0.5$ & $6.6 \pm 0.3$ & $7.0 \pm 0.3$ & $6.7 \pm 0.7$ \\
\hline Albumin (g/dL) & $2.8 \pm 0.3$ & $2.7 \pm 0.1$ & $2.9 \pm 0.2$ & $2.7 \pm 0.4$ \\
\hline $\mathrm{A} / \mathrm{G}$ ratio & $0.71 \pm 0.05$ & $0.69 \pm 0.03$ & $0.71 \pm 0.08$ & $0.69 \pm 0.07$ \\
\hline Total bilirubin (mg/dL) & $0.09 \pm 0.05$ & $0.08 \pm 0.03$ & $0.06 \pm 0.02$ & $0.03 \pm 0.01$ \\
\hline $\mathrm{LDH}(\mathrm{IU} / \mathrm{L})$ & $600 \pm 476$ & $579 \pm 353$ & $420 \pm 327$ & $481 \pm 426$ \\
\hline Cholesterol (mg/dL) & $85 \pm 18$ & $72 \pm 16$ & $91 \pm 23$ & $84 \pm 20$ \\
\hline Triglycerides (mg/dL) & $23 \pm 11$ & $19 \pm 7$ & $31 \pm 15$ & $22 \pm 11$ \\
\hline $\mathrm{AST}(\mathrm{U} / \mathrm{L})$ & $113 \pm 43$ & $103 \pm 33$ & $87 \pm 16$ & $90 \pm 15$ \\
\hline $\operatorname{ALT}(\mathrm{U} / \mathrm{L})$ & $42 \pm 23$ & $36 \pm 22$ & $32 \pm 8$ & $29 \pm 5$ \\
\hline ALP (U/L) & $140 \pm 34$ & $163 \pm 38$ & $130 \pm 37$ & $174 \pm 50$ \\
\hline $\operatorname{Mg}(\mathrm{mg} / \mathrm{dL})$ & $2.9 \pm 0.1$ & $2.8 \pm 0.1$ & $2.7 \pm 0.2$ & $2.8 \pm 0.2$ \\
\hline $\mathrm{Ca}(\mathrm{mg} / \mathrm{dL})$ & $9.9 \pm 0.2$ & $9.8 \pm 0.2$ & $10.0 \pm 0.4$ & $10.5 \pm 0.5$ * \\
\hline $\mathrm{Na}(\mathrm{mmol} / \mathrm{L})$ & $134 \pm 2$ & $135 \pm 5$ & $136 \pm 3$ & $142 \pm 1^{* *}$ \\
\hline $\mathrm{K}(\mathrm{mmol} / \mathrm{L})$ & $5.8 \pm 0.7$ & $6.1 \pm 0.2$ & $6.2 \pm 0.2$ & $6.0 \pm 0.3$ \\
\hline
\end{tabular}

Data are presented as mean \pm standard deviation; ${ }^{*}$ Significantly different from corresponding control value at $p<0.05 ; * *$ Significantly different from corresponding control value at $p<0.01$; RCMLS: Rubus coreanus Miquel leaves and stems, ALP: alkaline phosphatase, ALT: alanine transaminase; AST: aspartate transaminase, A/G ratio: Albumin/Globulin ratio, $\mathrm{LDH}$ : lactate dehydrogenase, BUN: blood urea nitrogenm Mg: magnesium, Ca: calcium, Na: sodium, K: potassium. 
Table 8. Serum biochemistry in 13 weeks repeated oral dose toxicity study of the leaves and stems of Rubus coreanus Miquel.

\begin{tabular}{|c|c|c|c|c|}
\hline Parameter & Control & $\begin{array}{l}625 \mathrm{mg} / \mathrm{kg} \\
\mathrm{RCMLS}\end{array}$ & $\begin{array}{l}1250 \mathrm{mg} / \mathrm{kg} \\
\mathrm{RCMLS}\end{array}$ & $\begin{array}{c}2500 \mathrm{mg} / \mathrm{kg} \\
\text { RCMLS }\end{array}$ \\
\hline \multicolumn{5}{|l|}{ Male } \\
\hline Glucose (mg/dL) & $184 \pm 34$ & $198 \pm 36$ & $193 \pm 23$ & $197 \pm 26$ \\
\hline BUN (mg/dL) & $14.8 \pm 2.0$ & $14.2 \pm 2.4$ & $13.9 \pm 2.8$ & $14.7 \pm 1.7$ \\
\hline Creatinine $(\mathrm{mg} / \mathrm{dL})$ & $0.58 \pm 0.11$ & $0.60 \pm 0.13$ & $0.59 \pm 0.11$ & $0.53 \pm 0.13$ \\
\hline Total protein $(\mathrm{g} / \mathrm{dL})$ & $6.7 \pm 0.5$ & $6.6 \pm 0.3$ & $7.0 \pm 0.3$ & $6.7 \pm 0.7$ \\
\hline Albumin (g/dL) & $2.8 \pm 0.3$ & $2.7 \pm 0.1$ & $2.9 \pm 0.2$ & $2.7 \pm 0.4$ \\
\hline $\mathrm{A} / \mathrm{G}$ ratio & $0.71 \pm 0.05$ & $0.69 \pm 0.03$ & $0.71 \pm 0.08$ & $0.69 \pm 0.07$ \\
\hline Total bilirubin (mg/dL) & $0.09 \pm 0.05$ & $0.08 \pm 0.03$ & $0.06 \pm 0.02$ & $0.03 \pm 0.01$ \\
\hline $\mathrm{LDH}(\mathrm{IU} / \mathrm{L})$ & $600 \pm 476$ & $579 \pm 353$ & $420 \pm 327$ & $481 \pm 426$ \\
\hline Cholesterol (mg/dL) & $85 \pm 18$ & $72 \pm 16$ & $91 \pm 23$ & $84 \pm 20$ \\
\hline Triglycerides (mg/dL) & $23 \pm 11$ & $19 \pm 7$ & $31 \pm 15$ & $22 \pm 11$ \\
\hline $\operatorname{AST}(\mathrm{U} / \mathrm{L})$ & $113 \pm 43$ & $103 \pm 33$ & $87 \pm 16$ & $90 \pm 15$ \\
\hline $\operatorname{ALT}(\mathrm{U} / \mathrm{L})$ & $42 \pm 23$ & $36 \pm 22$ & $32 \pm 8$ & $29 \pm 5$ \\
\hline $\operatorname{ALP}(\mathrm{U} / \mathrm{L})$ & $140 \pm 34$ & $163 \pm 38$ & $130 \pm 37$ & $174 \pm 50$ \\
\hline $\operatorname{Mg}(\mathrm{mg} / \mathrm{dL})$ & $2.6 \pm 0.2$ & $2.6 \pm 0.2$ & $2.5 \pm 0.2$ & $2.6 \pm 0.2$ \\
\hline $\mathrm{Ca}(\mathrm{mg} / \mathrm{dL})$ & $10.0 \pm 0.3$ & $10.0 \pm 0.2$ & $10.0 \pm 0.2$ & $10.0 \pm 0.3$ \\
\hline $\mathrm{Na}(\mathrm{mmol} / \mathrm{L})$ & $141 \pm 5$ & $143 \pm 5$ & $142 \pm 4$ & $142 \pm 4$ \\
\hline $\begin{array}{c}\mathrm{K}(\mathrm{mmol} / \mathrm{L}) \\
\text { Female }\end{array}$ & $5.4 \pm 0.7$ & $5.4 \pm 0.4$ & $5.5 \pm 0.4$ & $5.8 \pm 0.9$ \\
\hline Glucose (mg/dL) & $192 \pm 32$ & $212 \pm 17$ & $210 \pm 19$ & $206 \pm 22$ \\
\hline $\mathrm{BUN}(\mathrm{mg} / \mathrm{dL})$ & $13.8 \pm 1.7$ & $14.5 \pm 1.7$ & $13.3 \pm 1.8$ & $14.7 \pm 1.8$ \\
\hline Creatinine $(\mathrm{mg} / \mathrm{dL})$ & $0.44 \pm 0.04$ & $0.48 \pm 0.03$ & $0.46 \pm 0.03$ & $0.46 \pm 0.05$ \\
\hline Total protein $(\mathrm{g} / \mathrm{dL})$ & $6.3 \pm 0.3$ & $6.3 \pm 0.2$ & $6.3 \pm 0.3$ & $6.2 \pm 0.4$ \\
\hline Albumin (g/dL) & $2.3 \pm 0.1$ & $2.3 \pm 0.1$ & $2.3 \pm 0.1$ & $2.3 \pm 0.1$ \\
\hline $\mathrm{A} / \mathrm{G}$ ratio & $0.56 \pm 0.04$ & $0.57 \pm 0.03$ & $0.58 \pm 0.05$ & $0.59 \pm 0.03$ \\
\hline Total bilirubin (mg/dL) & $0.03 \pm 0.05$ & $0.04 \pm 0.02$ & $0.02 \pm 0.02$ & $0.03 \pm 0.02$ \\
\hline $\mathrm{LDH}(\mathrm{IU} / \mathrm{L})$ & $649 \pm 619$ & $944 \pm 526$ & $763 \pm 544$ & $646 \pm 676$ \\
\hline Cholesterol (mg/dL) & $69 \pm 24$ & $67 \pm 15$ & $73 \pm 15$ & $65 \pm 11$ \\
\hline Triglycerides (mg/dL) & $35 \pm 15$ & $39 \pm 21$ & $47 \pm 19$ & $32 \pm 15$ \\
\hline $\operatorname{AST}(\mathrm{U} / \mathrm{L})$ & $109 \pm 28$ & $119 \pm 29$ & $109 \pm 30$ & $105 \pm 26$ \\
\hline $\operatorname{ALT}(\mathrm{U} / \mathrm{L})$ & $39 \pm 9$ & $39 \pm 7$ & $35 \pm 6$ & $37 \pm 7$ \\
\hline ALP (U/L) & $225 \pm 35$ & $225 \pm 44$ & $195 \pm 39$ & $234 \pm 44$ \\
\hline $\operatorname{Mg}(\mathrm{mg} / \mathrm{dL})$ & $2.7 \pm 0.1$ & $2.7 \pm 0.2$ & $2.5 \pm 0.2$ & $2.5 \pm 0.1 *$ \\
\hline $\mathrm{Ca}(\mathrm{mg} / \mathrm{dL})$ & $10.2 \pm 0.8$ & $10.1 \pm 0.4$ & $10.5 \pm 0.6$ & $10.2 \pm 0.5$ \\
\hline $\mathrm{Na}(\mathrm{mmol} / \mathrm{L})$ & $140 \pm 2$ & $138 \pm 3$ & $136^{* *} \pm 3$ & $135 \pm 4^{* *}$ \\
\hline $\mathrm{K}(\mathrm{mmol} / \mathrm{L})$ & $5.7 \pm 0.9$ & $5.4 \pm 0.5$ & $5.6 \pm 0.7$ & $5.9 \pm 0.4$ \\
\hline
\end{tabular}

Data are presented as mean \pm standard deviation; * Significantly different from corresponding control value at $p<0.05 ; * *$ Significantly different from corresponding control value at $p<0.01$; RCMLS: Rubus coreanus Miquel leaves and stems, ALP: alkaline phosphatase, ALT: alanine transaminase; AST: aspartate transaminase, A/G ratio: Albumin/Globulin ratio, $\mathrm{LDH}$ : lactate dehydrogenase, BUN: blood urea nitrogenm Mg: magnesium, Ca: calcium, Na: sodium, K: potassium. 
Table 9. Absolute organ weights of male and female rats in 14 days repeated oral dose and 13 weeks repeated oral dose toxicity studies of the leaves and stems of Rubus coreanus Miquel.

\begin{tabular}{|c|c|c|c|c|c|c|c|c|c|c|c|c|c|}
\hline \multirow{3}{*}{ Group } & \multirow{3}{*}{ Sex } & \multicolumn{12}{|c|}{ Organ Weight (mg/kg/Day) } \\
\hline & & \multicolumn{2}{|c|}{ Liver } & \multicolumn{2}{|c|}{ Adrenal (Left) } & \multicolumn{2}{|c|}{ Adrenal (Right) } & \multicolumn{2}{|c|}{ Testis/Ovary (Left) } & \multicolumn{2}{|c|}{ Testis/Ovary (Right) } & \multicolumn{2}{|c|}{ Spleen } \\
\hline & & 14 Days & 13 Weeks & 14 Days & 13 Weeks & 14 Days & 13 Weeks & 14 Days & 13 Weeks & 14 Days & 13 Weeks & 14 Days & 13 Week \\
\hline Control & $\begin{array}{c}\text { Male } \\
\text { Female }\end{array}$ & $\begin{array}{c}8.8070 \pm \\
0.6145 \\
6.4639 \pm \\
1.1599\end{array}$ & $\begin{array}{c}14.4278 \pm \\
2.2296 \\
7.6186 \pm \\
0.9044\end{array}$ & $\begin{array}{c}0.0275 \pm \\
0.0033 \\
0.0336 \pm \\
0.0042\end{array}$ & $\begin{array}{c}0.0329 \pm \\
0.0065 \\
0.0353 \pm \\
0.0054\end{array}$ & $\begin{array}{c}0.0247 \pm \\
0.0018 \\
0.0275 \pm \\
0.0049\end{array}$ & $\begin{array}{c}0.0307 \pm \\
0.0082 \\
0.0362 \pm \\
0.0055\end{array}$ & $\begin{array}{c}1.4477 \pm \\
0.1851 \\
0.0504 \pm \\
0.0151\end{array}$ & $\begin{array}{c}1.9374 \pm \\
0.1253 \\
0.0445 \pm \\
0.0089\end{array}$ & $\begin{array}{c}.4947 \pm \\
0.1887 \\
0.0431 \pm \\
0.0120\end{array}$ & $\begin{array}{c}1.9352 \pm \\
0.1137 \\
0.0480 \pm \\
0.0088\end{array}$ & $\begin{array}{c}0.7522 \pm \\
0.1046 \\
0.4818 \pm \\
0.0517\end{array}$ & $\begin{array}{c}.0541 \pm \\
0.1852 \\
0.5485 \pm \\
0.0661\end{array}$ \\
\hline $\begin{array}{c}625 \mathrm{mg} / \mathrm{kg} \\
\text { RCMLS }\end{array}$ & $\begin{array}{c}\text { Male } \\
\text { Female }\end{array}$ & $\begin{array}{c}9.5767 \pm \\
0.9401 \pm \\
6.1001 \pm \\
0.2304\end{array}$ & $\begin{array}{c}14.5220 \pm \\
1.4821 \pm \\
7.3090 \pm \\
1.0428\end{array}$ & $\begin{array}{c}0.0263 \pm \\
0.0048 \\
0.0322 \pm \\
0.0049\end{array}$ & $\begin{array}{c}0.0300 \pm \\
0.0055 \\
0.0363 \pm \\
0.0086\end{array}$ & $\begin{array}{c}0.0242 \pm \\
0.0040 \\
0.0305 \pm \\
0.0049\end{array}$ & $\begin{array}{c}0.0275 \pm \\
0.0046 \\
0.0331 \pm \\
0.0057\end{array}$ & $\begin{array}{c}1.4641 \pm \\
0.0960 \\
0.0418 \pm \\
0.0052\end{array}$ & $\begin{array}{c}1.8488 \pm \\
0.1465 \\
0.0470 \pm \\
0.0099\end{array}$ & $\begin{array}{c}1.4779 \pm \\
0.0567 \\
0.0389 \pm \\
0.0065\end{array}$ & $\begin{array}{c}1.8611 \pm \\
0.1518 \\
0.0475 \pm \\
0.0068\end{array}$ & $\begin{array}{c}0.6861 \pm \\
0.0548 \\
0.4437 \pm \\
0.0598\end{array}$ & $\begin{array}{c}1.0005 \pm \\
0.1286 \\
0.6002 \pm \\
0.0768\end{array}$ \\
\hline $\begin{array}{c}1250 \mathrm{mg} / \mathrm{kg} \\
\text { RCMLS }\end{array}$ & $\begin{array}{c}\text { Male } \\
\text { Female }\end{array}$ & $\begin{array}{c}8.3576 \pm \\
0.4890 \\
6.4504 \pm \\
0.8888\end{array}$ & $\begin{array}{c}15.239 \pm \\
1.7837 \\
7.6644 \pm \\
0.7581\end{array}$ & $\begin{array}{c}0.0221 \pm \\
0.0044 \\
0.0343 \pm \\
0.0054\end{array}$ & $\begin{array}{c}0.0270 \pm \\
0.0074 \\
0.0329 \pm \\
0.0067\end{array}$ & $\begin{array}{c}0.0205 \pm \\
0.0049 \\
0.0332 \pm \\
0.0054\end{array}$ & $\begin{array}{c}0.0284 \pm \\
0.0042 \\
0.0329 \pm \\
0.0051\end{array}$ & $\begin{array}{c}1.2772 \pm \\
0.3645 \\
0.0456 \pm \\
0.0119\end{array}$ & $\begin{array}{c}1.8611 \pm \\
0.1288 \\
0.0487 \pm \\
0.0087\end{array}$ & $\begin{array}{c}1.2739 \pm \\
0.3564 \\
0.0395 \pm \\
0.0026\end{array}$ & $\begin{array}{c}1.8696 \pm \\
0.1363 \\
0.0452 \pm \\
0.0060\end{array}$ & $\begin{array}{c}0.6647 \pm \\
0.1423 \\
0.4856 \pm \\
0.0703\end{array}$ & $\begin{array}{c}0.9661 \pm \\
0.1545 \\
0.5770 \pm \\
0.1031\end{array}$ \\
\hline $\begin{array}{c}2500 \mathrm{mg} / \mathrm{kg} \\
\text { RCMLS }\end{array}$ & $\begin{array}{c}\text { Male } \\
\text { Female }\end{array}$ & $\begin{array}{c}8.5526 \pm \\
0.70344 \\
6.2359 \pm \\
0.5127\end{array}$ & $\begin{array}{c}12.5166 \pm \\
1.63511^{*} \\
6.9086 \pm \\
0.8667\end{array}$ & $\begin{array}{c}0.0250 \pm \\
0.0073 \\
0.0330 \pm \\
0.0028\end{array}$ & $\begin{array}{c}0.0296 \pm \\
0.0042 \\
0.0316 \pm \\
0.0032\end{array}$ & $\begin{array}{c}0.0253 \pm \\
0.0065 \\
0.0311 \pm \\
0.0035\end{array}$ & $\begin{array}{c}0.0291 \pm \\
0.0044 \\
0.0308 \pm \\
0.0038\end{array}$ & $\begin{array}{c}1.3956 \pm \\
0.1045 \\
0.0409 \pm \\
0.0041\end{array}$ & $\begin{array}{c}1.8965 \pm \\
0.0922 \\
0.0368 \pm \\
0.0101\end{array}$ & $\begin{array}{c}1.3873 \pm \\
0.1331 \\
0.0411 \pm \\
0.0092\end{array}$ & $\begin{array}{c}1.9431 \pm \\
0.0692 \\
0.0395 * \pm \\
0.0061\end{array}$ & $\begin{array}{c}0.7834 \pm \\
0.2388 \\
0.5028 \pm \\
0.0881\end{array}$ & $\begin{array}{c}0.8626 \pm \\
0.1287 \\
0.5510 \pm \\
0.0585\end{array}$ \\
\hline
\end{tabular}

Data are presented as mean \pm standard deviation; * Significantly different from corresponding control value at $p<0.05$; RCMLS: Rubus coreanus Miquel leaves and stems.

Table 10. Relative organ weights of male and female rats in 14 days repeated oral dose and 13 weeks repeated oral dose toxicity studies of the leaves and stems of

Rubus coreanus Miquel.

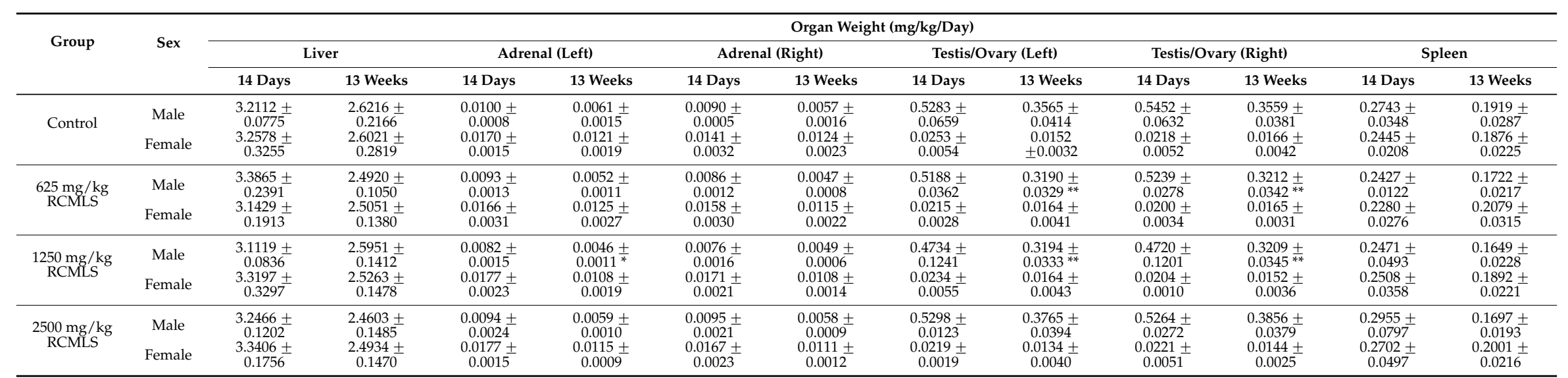

Data are presented as mean \pm standard deviation; * Significantly different from corresponding control value at $p<0.05 ;{ }^{* *}$ Significantly different from corresponding control value at

$p<0.01$; RCMLS: Rubus coreanus Miquel leaves and stems. 
However, it was slight and within the biological fluctuation range and did not show dose-dependency. Additionally, no clear relative change in absolute and relative organ weight was found. Therefore, it was not considered toxicologically significant. At the histopathology test, in a male of the vehicle control group, the cyst observed in the pituitary gland was confirmed as a nerve cyst. Also, the mass observed in the subcutaneous dorsal neck in a female of the $625 \mathrm{mg} / \mathrm{kg} /$ day group was confirmed as an adenocarcinoma in the mammary gland. However, no metastasis findings were observed, there was no dose-dependency, and they are sometimes naturally observed in the female mammary gland.

The histopathology test of the lungs showed inflammation of the terminal bronchiole and alveolar duct and foreign body granuloma. Inflammation was found to be somewhat dose-dependent in both the male and female test substance administered groups. However, the vehicle control group also showed inflammation. Considering that it was found near terminal bronchiole and alveolar duct, and the foreign body granuloma covered in sporadic brown foreign substance was found in some individuals, and administered dosage was at $20 \mathrm{~mL} / \mathrm{kg}$, which was quite high and formed much froth on preparation, the findings were evaluated to have caused by aspiration during administration. Therefore, it does not seem to be a side effect of RCMLS consumption. Including the osseous metaplasia of the lungs found in a male of the vehicle control group, the rest of the female and male of the vehicle control group, and $2500 \mathrm{mg} / \mathrm{kg} /$ day group findings were either observed in the control group, were sporadic, or were natural occurrences. Therefore, it was not considered toxicologically significant.

In conclusion, as the result of repeated 13 weeks oral administration of RCMLS, no systemic toxicological changes were observed. Therefore, the no observed adverse effect level (NOAEL) of RCMLS was set at $2500 \mathrm{mg} / \mathrm{kg} /$ day and no target organs were observed.

\section{Experimental Section}

\subsection{Preparation of the Leaves and Stems of Rubus coreanus Miquel}

RCMLS was collected in May 2014 from the Gochang Bokbunja Research Institute, Gochang, South Korea. The dried leaves and stems were mixed in the same percentage and pulverized using a Pin-type Mill (Seichin, Tokyo, Japan). The pulverized sample was stored at $4{ }^{\circ} \mathrm{C}$ prior to use. RCMLS was prepared by suspending the powder with sterile water for injection according to the doses assigned to each group in the single, 14 days and 13 weeks repeated oral dose toxicity studies.

\subsection{Nutrition Composition}

Proximate composition analyses of RCMLS were done for moisture, ash, crude protein, crude fat, carbohydrate and fiber contents according to the methods described by the Korean Food Standards Codex [29] and Korean Health Functional Food Code [30]. Moisture was determined by oven-drying at $105{ }^{\circ} \mathrm{C}$ to constant weight. Ash content was determined gravimetrically in a muffle furnace by heating at $550{ }^{\circ} \mathrm{C}$ to constant weight. Crude fat content was determined using the decomposition method. Crude protein $(6 \times 6.25)$ was determined on a LECO ${ }^{\circledR} \operatorname{TruSpec}^{\circledR} \mathrm{CN}$ (Carbon/Nitrogen Determinator, St. Joseph, MI, USA). Carbohydrate content was calculated as the difference between 100 and the sum of the moisture, crude protein, crude fat and ash contents. The dietary fiber content was obtained as an indigestible residue after enzymatic digestion of non-dietary fiber components.

Mineral, vitamin contents and fatty acid composition of RCMLS were done according to the methods described by the Korean Health Functional Food Code using inductive coupled plasma (ICP, Teledyne Leeman Labs, Hudson, NH, USA), Nanospace SI-2 HPLC system (Shiseido Co., Ltd., Tokyo, Japan) and Agilent 7890 GC system (Agilent Technologies, Santa Clara, CA, USA), respectively.

\subsection{Experimental Animals}

Male and female specific pathogene free SD rats (160-220 g) were obtained from the animal facility of Orient Bio (Sungnam, Korea). In case of the females, only ones that were not pregnant or 
had ever given birth were selected. The rats used in this study are widely used in micronuclear tests, as it is a suitable test animal in toxicology experiments. Also this strain of rat has much accumulated base test data, which can be used in test result interpretation and evaluation. The animals were starved, with exception to water, from the night before the forced oral administration using a probe to empty stomach contents. After $3-4 \mathrm{~h}$ food was redistributed. The animals were housed in a room maintained at a temperature of $23.2 \pm 0.3^{\circ} \mathrm{C}$ and $23.2 \pm 0.8^{\circ} \mathrm{C}$, and a relative humidity of $51.4 \% \pm 3.4 \%$ and $51.4 \% \pm 5.4 \%$ for the 14 days single dose and repeated dose toxicity, respectively. The animals were housed in a room maintained at a temperature of $22.9 \pm 0.7^{\circ} \mathrm{C}$ and a relative humidity of $47.9 \% \pm 3.9 \%$ during the 13 weeks repeated dose toxicity study. They had a $12 \mathrm{~h}$ dark/light cycle. Procedures involving animals and their care were approved by the ethics committee, Korea Conformity Laboratories, 2014.

\subsection{Study Design Overview}

This study was conducted in compliance with the Good Laboratory Practice (GLP) and Test Guidelines of the Organization for Economic Cooperation and Development (OECD) [31,32], and the Korea Food and Drug Administration (KDFA) at the GLP institute approved by the KFDA [33,34].

\subsubsection{Single Oral dose Toxicity Study}

SD rats of both male and female were divided into two groups (I, II) of 22 rats (11 males and 11 females) matched for weight. All animals had free access to water and food throughout the experimental period. Group II was administrated RCMLS at single oral doses of the $2500 \mathrm{mg} / \mathrm{kg}$, and Group I served as the vehicle control, receiving the same volume of sterile water. Animals were starved, with exception to water, from the night before the forced oral administration using a probe to empty stomach contents, and food was offered approximately $4 \mathrm{~h}$ after administration. Dosing volume was set to $20 \mathrm{~mL} / \mathrm{kg}$ for toxicity study, based on the most recent body weight.

Clinical signs of animals were continuously monitored during the first $24 \mathrm{~h}$ and then daily thereafter, for a total period of 14 days. Changes in the normal activity of rats were monitored and the time at which signs of toxicity or death appeared was documented. On administration day, test animals were observed after $30 \mathrm{~min}$ of administration and every $6 \mathrm{~h}$ thereafter. Clinical signs observation was conducted until 14 days after administration. Changes in the body weights of the rats were monitored. At the end of the observation period, all surviving animals were fasted overnight.

After 14 days of administration, all surviving rats were anesthetized with $\mathrm{CO}_{2}$ gas to open their abdomen. Then they were killed by severing the caudal vena cava and the abdominal artery for bloodletting to observe the organs with the naked eye.

\subsubsection{Days Repeated Oral dose Toxicity Study}

Four groups of 22 animals (11 males and 11 females) were used. Groups II-IV received RCMLS at daily doses of $625 \mathrm{mg} / \mathrm{kg} /$ day, $1250 \mathrm{mg} / \mathrm{kg} /$ day and $2500 \mathrm{mg} / \mathrm{kg} /$ day, and Group I served as the vehicle control receiving the same volume of distilled water, for a period of 14 days. Animals underwent forced oral administration using a probe to empty stomach contents. Dosing volume was set to $20 \mathrm{~mL} / \mathrm{kg}$ / day for the toxicity study, based on the most recent body weight. During the period of administration, the animals were weighed and observed daily to detect any clinical signs of toxicity. After 14 days, all surviving animals were investigated in the same way used in the single oral dose toxicity study.

Additionally, food consumption measurements were taken at initial administration and once weekly. Method of measurement was, food distribution amount on the day before weigh-in was recorded and then the remaining food amount on the day of weigh-in was used to calculate 1 day food consumption amount. Average consumption volume (g/rat/day) was calculated per individual animal. Appearance of animals' eyes was observed at group division and on the last week of administration 
precedents of the vehicle control group and high dosage group (Group IV, $2500 \mathrm{mg} / \mathrm{kg} /$ day) were observed by the naked eye.

During the last administration week, five rats from each test group were transferred to a metabolic cage, where fresh urine was collected and tested for following items using urine screening test (Siemens) and urine auto-analyzer (CliniTek 50, Siemens/Bayer, Munich, Germany; MAI-050-01). Urine tone was observed with the naked eye.

Hematology test was conducted using a blood analyzer (ADVIA 2120, Siemens; MAI-105-01). Planned autopsy rats were starved overnight, anesthetized with $\mathrm{CO}_{2}$ gas, then blood was collected from ventral aorta through laparoscopy. EDTA-2K anticoagulant was used.

For serum biochemistry test, blood chemical testing device (Hitachi 7180, Hitachi, Tokyo, Japan; MAI-059-01) was used. The serum used derived from the blood collected from abdominal aorta at planned autopsy was centrifuged for $10 \mathrm{~min}$ at $3000 \mathrm{rpm}$.

After the last administration and autopsy, the following organs were extracted and weighed on an electronic scale. All following organs listed below were fixed with $10 \%$ sterile formalin solution: thymus, spleen, pancreas, stomach, duodenum, jejunum, ileum, cecum, colon, rectum, mesenteric lymph nodes, mandibular lymph nodes, salivary gland, thyroid gland (including parathyroid gland), Harderian gland, heart, lung, kidneys, adrenal glands, liver, aorta, brain, pituitary gland, tongue, trachea, esophagus, sternum, thoracic spinal cord, femorotibial joint, peripheral nerve (sciatic), skeletal muscle (femoral), prostate gland, seminal vesicles, ovaries, uterus, vagina, urinary bladder, and skin (including mammary gland). The testes were fixed in Bouin's solution and the eyes in Davidson's solution.

\subsubsection{Weeks Repeated Oral dose Toxicity Study}

Four groups of 88 rats ( 44 males and 44 females) were used. Groups II-IV received RCMLS at daily doses of 625,1250 and $2500 \mathrm{mg} / \mathrm{kg} /$ day, and Group I served as the vehicle control receiving distilled water at the same volume, for a period of 13 weeks. During the period of administration, the animals were weighed and observed daily to detect any signs of toxicity. After 13 weeks, all surviving animals were investigated in the same way that was used in the subchronic toxicity study of 14 days repeated oral dose.

\subsection{Statistical Analysis}

Data are expressed as the means \pm standard deviation and analyzed by a one-way analysis of variance (ANOVA). When significant differences existed, Duncan's multiple range test was used to compare the means. SPSS $12.0 \mathrm{~K}$ (SPSS Inc., Chicago, IL, USA) was used for all statistical analysis. A $p<0.05$ or 0.01 were considered as statistically different.

\section{Conclusions}

To investigate the possible application of RCMLS as food materials, we carried out an analysis of the nutritional composition and toxicity of RCMLS following 14 days single and repeated oral administration and 13 weeks of repeated oral administration to SD rats. The proximate composition of RCMLS was $76.81 \%$ carbohydrate, $10.07 \%$ crude protein, $5.57 \%$ moisture, $5.17 \%$ crude ash and $2.20 \%$ crude fat, respectively. RCMLS contained high contents of minerals and vitamins such as K and E, respectively. The major fatty acid components were $30.48 \% \alpha$-linolenic acid, $23.68 \%$ palmitic acid and $15.51 \%$ linoleic acid. Single and repeated oral administration of RCMLS produced no significant toxic effects in SD rats. Under the present experimental conditions, the approximate $\mathrm{LD}_{50}$ of RCMLS might be over $2500 \mathrm{mg} / \mathrm{kg}$ b.w for both male and female, the NOAEL might be over $2500 \mathrm{mg} / \mathrm{kg} /$ day for both male and female, and no target organs were identified.

Acknowledgments: This study was conducted by Rural Development Administration (RDA) Cooperative Research (Project number: PJ010501022015) support. We thank Kelly Kim for English translation and proofreading. 
Author Contributions: A.S.O., H.R.K. and J.S.C. designed research. A.S.O., Y.N.S. and G.M.N. analyzed data. A.S.O. and Y.N.S. wrote the manuscript.

Conflicts of Interest: The authors declare no conflict of interest.

\section{References}

1. Bae, G.H. The Medicinal Plants of Korea; Kyohak Publishing Co.: Seoul, Korea, 2000; p. 231.

2. Lee, Y.; Kim, J.C.; Hwang, K.T.; Kim, D.H.; Jung, C.M. Quality characteristics of black raspberry wine fermented with different yeasts. J. Korean Soc. Food Sci. Nutr. 2013, 42, 784-791. [CrossRef]

3. Moon, G.S. Constituents and Uses of Medicinal Herbs; Ilweolseogak: Seoul, Korea, 1991; pp. 310-311.

4. Tulio, A.Z., Jr.; Reese, R.N.; Wyzgoski, F.J.; Rinaldi, P.L.; Fu, R.; Scheerens, J.C.; Miller, A. Cyanidin 3-rutinoside and cyanidin 3-xylosylrutinoside as primary phenolic antioxidants in black raspberry. J. Agric. Food Chem. 2008, 56, 1880-1888. [CrossRef] [PubMed]

5. Kim, Y.H.; Choi, J.H.; Rim, H.K.; Kang, H.J.; Chang, S.G.; Park, J.H.; Park, H.J.; Choi, J.W.; Kim, S.D.; Lee, K.T. 23-Hydroxytormentic acid and nigaichgoside $\mathrm{f}(1)$ isolated from Rubus coreanus attenuate cisplatin-induced cytotoxicity by reducing oxidative stress in renal epithelial LLC-PK(1) cells. Biol. Pharm. Bull. 2011, 34, 906-911. [CrossRef] [PubMed]

6. Lee, J.; Dossett, M.; Finn, C.E. Rubus fruit phenolic research: The good, the bad, and the confusing. Food Chem. 2012, 130, 785-796. [CrossRef]

7. Pang, K.C.; Kim, M.S.; Lee, M.W. Hydrolyzable tannins from the fruits of Rubus coreanum. Korean J. Pharmacogn. 1996, 27, 366-370.

8. Kim, H.S.; Park, S.J.; Hyun, S.H.; Yang, S.O.; Lee, J.; Auh, J.H.; Kim, J.H.; Cho, S.M.; Marriott, P.J.; Choi, H.K. Biochemical monitoring of black raspberry (Rubus coreanus Miquel) fruits according to maturation stage by 1H-NMR using multiple solvent systems. Food Res. Int. 2011, 44, 1977-1988. [CrossRef]

9. Kim, K.H.; Lee, Y.A.; Kim, J.S.; Lee, D.I.; Cho, M.W.; Kim, D.H.; Jung, C.M. Antioxidative Activity of Tannins from Rubus coreanum. Korean J. Pharmacogn. 2000, 44, 354-357.

10. Jang, T.S.; Yang, G.C.; Im, S.Y.; Kim, B.B. Antioxidant and Antihemolytic Activity of Ethanol Extracts of Rubus coreanus Miquel. J. Korean Oil Chem. Soc. 2014, 31, 130-135. [CrossRef]

11. Kim, J.M. Characteristics of Rubus coreanus Miq. Fruits at different ripening stages. Korean J. Food Sci. Technol. 2011, 43, 341-347. [CrossRef]

12. Park, Y.K.; Choi, S.H.; Kim, S.H.; Han, J.G.; Chung, H.G. Changes in antioxidant activity, total phenolics and vitamin C content during fruit ripening in Rubus occidentalis. Korean J. Plant Res. 2007, 20, 461-465.

13. Baek, E.Y.; Lee, S.M.; Lee, J.E.; Park, E.K.; Kim, Y.R.; Jung, I.K.; Kim, J.H. Effect of Rubus coreanus Miquel on prostate tumour growth. J. Funct. Foods 2013, 5, 1478-1486. [CrossRef]

14. Kim, H.J.; Kang, K.J. Including Effects of Rubus coreanus on Cell Death and Apoptotic Gene Expressions in Human Breast Cancer Cells. J. East Asian Soc. Diet. Life 2013, 23, 723-732.

15. Jeon, Y.H.; Choi, S.W.; Kim, M.R. Antimutagenic and cytotoxic activity of ethanol and water extracts from Rubus coreanum. Korean J. Food Cook. Sci. 2009, 25, 379-386.

16. Kim, S.K.; Kim, H.; Kim, S.A.; Park, H.K.; Kim, W. Anti-inflammatory and anti-superbacterial activity of polyphenols isolated from black raspberry. Korean J. Physiol. Pharmacol. 2013, 17, 73-79. [CrossRef] [PubMed]

17. Chung, T.H.; Kim, J.C.; Lee, C.Y.; Moon, M.K.; Chae, S.C.; Lee, I.S.; Kim, S.H.; Hahn, K.S.; Lee, I.P. Potential antiviral effects of Terminalia chebula, Sanguisorba officinalis, Rubus coreanus and Rheum palmatum against duck hepatitis B virus (DHBV). Phytother. Res. 1997, 11, 179-182. [CrossRef]

18. Jung, K.A.; Han, D.; Kwon, E.K.; Lee, C.H.; Kim, Y.E. Antifatigue effect of Rubus coreanus Miquel extract in mice. J. Med. Food 2007, 10, 689-693. [CrossRef] [PubMed]

19. Forbes-Hernández, T.Y.; Giampieri, F.; Gasparrini, M.; Mazzoni, L.; Quiles, J.L.; Alvarez-Suarez, J.M.; Battino, M. The effects of bioactive compounds from plant foods on mitochondrial function: A focus on apoptotic mechanisms. Food Chem. Toxicol. 2014, 68, 154-182. [CrossRef] [PubMed]

20. Lee, M.W. Phenolic Compounds from the Leaves of Rubus coreanum. Korean J. Pharmacogn. 1995, 39, 200-204.

21. Lee, Y.A.; Lee, L.M. Tannins from Rubus coreanum. Korean J. Pharmacogn. 1995, 26, 27-30. 
22. Lee, M.J.; Lee, S.J.; Choi, H.R.; Lee, J.H.; Kwon, J.W.; Chae, K.S.; Jeong, J.T.; Lee, T.B. Improvement of Cholesterol and Blood Pressure in Fruit, Leaf and Stem Extracts from Black Raspberry in vitro. Korean J. Med. Crop Sci. 2014, 22, 177-187. [CrossRef]

23. Ministry of Agriculture and Forestry (MAF). Development of Immuno-Stimulators Using Extracts from the Leaves of Panax Ginseng C.A. Meyer; Ministry of Agriculture and Forestry: Sejong, Korea, 2002; pp. 182-197.

24. Jung, S.H.; Che, G.S.; Park, S.E.; Park, H.J.; Gwon, J.W.; Lee, H.G.; Jung, J.T.; Lee, J.C. Component Analysis and Antioxidant Activity of leaf and stem of Rubus coreanus Miquel as by-product. In Proceedings of the Spring Conference, Chung-Ang University, Seoul, Korea, 31 May 2013; Korea Society of Food \& Cookery Science: Seoul, Korea.

25. Lee, K.I.; Kim, S.M.; Kim, S.M.; Pyo, B.S. Comparison of Fatty Acids and Antibacterial Activity against Pathogen of Acne in Different Parts of Ripened Black Raspberry (Rubus coreanus Miquel). J. Korean Soc. Food Sci. Nutr. 2011, 40, 466-469. [CrossRef]

26. Petterino, C.; Argentino-Storino, A. Clinical chemistry and hematology historical data in control Sprague-Dawley rats from pre-clinical toxicity studies. Exp. Toxicol. Pathol. 2006, 57, 213-219. [CrossRef] [PubMed]

27. Mukinda, J.T.; Eagles, P.F. Acute and sub-chronic oral toxicity profiles of the aqueous extract of Polygala fruticosa in female mice and rats. J. Ethnopharmacol. 2010, 128, 236-240. [CrossRef] [PubMed]

28. Liju, V.B.; Jeena, K.; Kuttan, R. Acute and subchronic toxicity as well as mutagenic evaluation of essential oil from turmeric (Curcuma longa L.). Food Chem. Toxicol. 2013, 53, 52-61. [CrossRef] [PubMed]

29. Korea Food \& Drug Administration. Food Standards Codex; Korean Foods Industry Association: Seoul, Korea, 2005.

30. Korean Food \& Drug Administration. Health Functional Food Code; Korean Foods Industry Association: Seoul, Korea, 2008.

31. OECD. Guidelines for Testing of Chemicals, Guideline 407, Repeated Dose 28-Day Oral Toxicity Study in Rodents; OECD: Paris, France, 1995.

32. OECD. Guidelines for Testing of Chemicals, Guideline 408, Repeated Dose 90-Day Oral Toxicity Study in Rodents; OECD: Paris, France, 1998.

33. Korean Food \& Drug Administration. Good Laboratory Practice Regulation for Non-Clinical Laboratory Studies (Notification No. 2014-136); Korean Food \& Drug Administration: Seoul, Korea, 2014.

34. Korean Food \& Drug Administration. The Standards of Toxicity Study for Medicinal Products (Notification No. 2014-136); Korean Food \& Drug Administration: Seoul, Korea, 2014.

Sample Availability: Not Available

(C) 2016 by the authors; licensee MDPI, Basel, Switzerland. This article is an open access article distributed under the terms and conditions of the Creative Commons by Attribution (CC-BY) license (http:/ / creativecommons.org/licenses/by/4.0/). 